TRANSACTIONS OF THE

AMERICAN MATHEMATICAL SOCIETY

Volume 358, Number 6, Pages 2735-2758

S 0002-9947(06)03843-8

Article electronically published on January 27, 2006

\title{
CONSTRUCTION AND PROPERTIES OF QUASI-LINEAR FUNCTIONALS
}

\author{
ØRJAN JOHANSEN AND ALF B. RUSTAD
}

\begin{abstract}
Quasi-linear functionals are shown to be uniformly continuous and decomposable into a difference of two quasi-integrals. A predual space for the quasi-linear functionals inducing the weak*-topology is given. General constructions of quasi-linear functionals by solid set-functions and q-functions are given.
\end{abstract}

\section{INTRODUCTION}

The theory of topological measures (formerly called quasi-measures) originated in [1] and was shown there to represent quasi-states (for definitions see the basic results section). The notion of a signed topological measure in a compact Hausdorff space was introduced in [6] and was shown there to represent bounded quasi-linear functionals. Basic properties such as continuity and decomposition of signed topological measures remained open problems. In this article we show that quasi-linear functionals are indeed uniformly continuous, countably additive, and they decompose into a difference of topological measures in so-called q-spaces. We also present a predual space for the quasi-linear functionals as a direct limit. This turns the topology of pointwise convergence into a weak*-topology.

Solid set-functions have become the main tool for constructing topological measures, hence it would be desirable to have a similar concept for signed topological measures. It turns out that this is possible, but the definition of a signed solid set-function is more complex than for the unsigned case. The q-functions were introduced in [4] as an alternative construction technique for topological measures; a discussion on signed q-functions is contained in the last section.

\section{BASIC DEFINITIONS AND RESULTS}

Throughout the article, we will let $X$ denote a compact Hausdorff space. We will let $\mathcal{O}(X)$ and $\mathcal{C}(X)$ denote the open and closed subsets of $X$, respectively. Furthermore, we put $\mathcal{A}(X)=\mathcal{O}(X) \cup \mathcal{C}(X)$. When there is no confusion concerning the space in question, we will omit the space from the notation.

Notation 1. Whenever $U \in \mathcal{O}$, we let $\lim _{C \in \mathcal{C}} f(C)$ denote the limit (if any) of the net $f(C)$ with index set $\{C \in \mathcal{C}: C \subset U\}$, ordered by inclusion. Conversely,

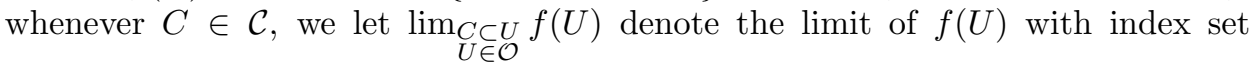

Received by the editors January 26, 2004 and, in revised form, August 30, 2004.

2000 Mathematics Subject Classification. Primary 28C15.

The first author was supported by the Norwegian Research Council.

(C)2006 American Mathematical Society Reverts to public domain 28 years from publication 2735 
$\{U \in \mathcal{O}: C \subset U\}$, ordered by reverse inclusion. (Here $f(C)$ may be any suitable expression in C.) Subfamilies of $\mathcal{C}$ and $\mathcal{O}$ may be specified to restrict the index set further.

Definition 2. A real valued function $\mu$ on $\mathcal{A}$ is called a signed topological measure if the following hold:

(1) If $A_{1}, \ldots, A_{n}, \biguplus_{i=1}^{n} A_{i} \in \mathcal{A}$, then $\mu\left(\biguplus_{i=1}^{n} A_{i}\right)=\sum_{i=1}^{n} \mu A_{i}$ (where $\biguplus$ indicates disjoint union).

(2) There is a constant $M<\infty$ such that whenever $A \in \mathcal{A},|\mu A| \leq M$.

(3) For any open set $U$

$$
\mu(U)=\lim _{\substack{C \subset U \\ C \in \mathcal{C}}} \mu(C) .
$$

Combining (1) and (2), we may also define

$$
\|\mu\|=\sup \left\{\sum_{i=1}^{n}\left|\mu A_{i}\right|:\left\{A_{i}\right\}_{i=1}^{n} \subset \mathcal{A} \text { disjoint }\right\} .
$$

Remark 3. Our definition is different from the definition in [6], but it is straightforward to prove that the two are equivalent. Our definition is however more convenient for the results presented here.

We denote the set of all signed topological measures in $X$ by $Q_{S}(X)$. The collection of (non-negative) topological measures will be denoted $Q(X)$.

The topological measures originated in [1]. It was proved in $[8$ that topological measures are countably additive. Their definition only differs from that of regular Borel measures by their domain of definition. Still, they are a vastly larger class of set functions with a rich mathematical structure.

The integral with respect to a signed topological measure is defined for all continuous functions $f \in C(X)$. Let $\mu \in Q_{S}(X), f \in C(X)$. Then defining $\mu_{f}(A)=\mu\left(f^{-1}(A)\right) ; A \in \mathcal{A}(\mathbb{R})$ yields a signed regular Borel measure $\mu_{f}$ in $\mathbb{R}$. (In general, such a combination with a continuous function maps topological measures to topological measures. However, in one-dimensional spaces it was shown in [7] that all signed topological measures extend uniquely to signed Borel measures.)

Definition 4. Let $\mu \in Q_{S}(X)$ and $f \in C(X)$. Then we define

$$
\mu(f)=\int x d \mu_{f}(x)
$$

where $\mu_{f}$ is the signed Borel measure given by $\mu_{f}(A)=\mu\left(f^{-1}(A)\right) ; A \in \mathcal{A}(\mathbb{R})$.

In the study of topological measures and their integrals the singly generated subalgebras of $C(X)$ play a crucial part. For $f \in C(X)$ let $A_{f}$ denote the uniformly closed subalgebra generated by $f$ and the constant functions. By the spectral theorem this algebra is isomorphic to the collection of continuous functions on the range of $f$.

Definition 5. A function $\rho: C(X) \rightarrow \mathbb{R}$ is called a (bounded) quasi-linear functional if it is a linear functional on $A_{f}$ for every $f \in C(X)$ and there is an $M<\infty$ such that $\rho(f) \leq M\|f\|_{\infty}$ for all $f \in C(X)$. We define $\|\rho\|$ as the smallest such $M$. If $\rho$ is positive (i.e. $\rho(f) \geq 0$ whenever $f \geq 0$ ) we call $\rho$ a quasi-integral. 
The quasi-linear functionals and signed topological measures have been shown to be in one-to-one isometric correspondence through the integral (cf. 6]). Accordingly we will not distinguish between the two and denote both by $Q_{S}(X)$. Similarly we will denote both the collection of quasi-integrals and the collection of topological measures by $Q(X)$.

Definition 6. Let $A$ be a subset of $X$. By a co-component of $A$ we mean a (connnected) component of $X \backslash A$. If $A$ has connected complement we call $A$ coconnected. If $A$ and its complement are both connected we will call the set solid. A restriction to solid sets will be denoted with a subscript $s$ (e.g. $\mathcal{C}_{s}$ will denote the compact solid sets). A restriction to connected sets will similarly be denoted with a subscript $c$.

The solid sets play an important role in the theory of topological measures. For many spaces they constitute a small and manageable family of sets which totally determines a topological measure. This is illustrated by the solid set-functions, which were introduced in 3 and whose properties were investigated there. In particular, the solid set-functions are invaluable tools for constructing topological measures.

Throughout the remainder of this section we will assume that $X$ is connected and locally connected. We include some definitions and results from [3] below:

Proposition 7. The following properties hold for $X$ :

(1) Let $K \in \mathcal{C}, U \in \mathcal{O}$ and $K \subset U$. If either $K$ or $U$ is connected, then there is a set $V \in \mathcal{O}_{c}$ such that $K \subset V \subset \bar{V} \subset U$.

(2) Let $K \in \mathcal{C}_{c}$. Then each connected component of $V=X \backslash K$ belongs to $\mathcal{O}_{s}$.

(3) Let $K \in \mathcal{C}_{s}, U \in \mathcal{O}$ and $K \subset U$. Then there is a set $V \in \mathcal{O}_{s}$ such that $K \subset V \subset \bar{V} \subset U$.

Definition 8. A partition of $X$ is a collection of mutually disjoint, non-void sets $\left\{A_{i}\right\}_{i \in I} \subset \mathcal{A}_{s}$, where at most finitely many of the sets $A_{i}$ are closed, and such that $X=\bigcup_{i \in I} A_{i}$. The number of closed sets in a partition $\mathcal{P}$ is called the order of $\mathcal{P}$. Partitions of order 1 (i.e. a set and its complement) are called trivial.

Definition 9. A partition $\left\{A_{i}\right\}_{i \in I}$ is irreducible if the following two conditions hold:

(1) $\bigcup_{i \in I, A_{i} \in \mathcal{C}} A_{i}$ is not co-connected.

(2) For any proper subset $I^{\prime} \subset\left\{i \in I: A_{i} \in \mathcal{C}\right\}, \bigcup_{i \in I^{\prime}} A_{i}$ is co-connected.

Definition 10. Let $n$ denote the maximal order of any irreducible partition of $X$. If $n$ is finite, define the genus of $X$ to be $g=n-1$. If $X$ only permits trivial partitions we call $X$ a $q$-space and set $g=0$.

Remark 11. The genus requirement was treated in [3] and [9]. When $X$ has genus zero, then a partition of $X$ into solid sets can have at most two members. This property is shared by a large class of spaces (e.g. when $X$ is simply connected).

Proposition 12. Let $\mathcal{F}=\left\{C_{j}\right\}_{j=1}^{n} \subset \mathcal{C}_{s}(n \geq 1)$ be a family of disjoint sets such that $\bigcup_{j=1}^{n} C_{j}$ is not co-connected. Then $\mathcal{F}$ has a subfamily $\mathcal{F}^{\prime}$ such that each connected component $U_{i}(i \in I)$ of $U=X \backslash \bigcup_{C_{j} \in \mathcal{F}^{\prime}} C_{j}$ belongs to $\mathcal{O}_{s}$, and $\mathcal{F}^{\prime} \cup$ $\left(\bigcup_{i \in I} U_{i}\right)$ is an irreducible partition of $X$. 
Definition 13. A function $\mu: \mathcal{A}_{s} \rightarrow \mathbb{R}^{+}$is a solid set-function if it satisfies

(1) For any finite collection of disjoint sets $\left\{C_{1}, \ldots, C_{n}\right\} \subset \mathcal{C}_{s}$ such that $C_{i} \subset$ $C \in \mathcal{C}_{s}$ for $i=1, \ldots, n$ we have

$$
\sum_{i=1}^{n} \mu C_{i} \leq \mu C
$$

(2) For all $U \in \mathcal{O}_{s}$ we have

$$
\mu(U)=\sup \left\{\mu(C): C \subset U, C \in \mathcal{C}_{s}\right\}
$$

(3) For any trivial or irreducible partition $\left\{A_{i}\right\}_{i \in I}$ of $X$ we have

$$
\mu(X)=\sum_{i \in I} \mu\left(A_{i}\right)
$$

Unlike in 3 ] we have not assumed $\mu(X)=1$.

The main construction result (Theorem 5.1 in [3]) states that solid set-functions extend uniquely to topological measures.

We include the following definition and proposition from [4] (again with the generalization that we do not assume $f(1)=1$ ):

Definition 14. A function $f:[0,1] \rightarrow \mathbb{R}^{+}$is called a $q$-function if it is continuous from the right and satisfies

(1) $f(0)=0, f(x-)+f(1-x)=f(1) ; x \in(0,1]$.

(2) $\sum_{i=1}^{n} f\left(x_{i}\right) \leq f\left(\sum_{i=1}^{n} x_{i}\right)$ whenever $x_{1}, x_{2}, \ldots, x_{n} \in[0,1]$ and $\sum_{i=1}^{n} x_{i}<1$.

Let $\nu$ be a normalized Borel (or topological) measure in $X$, i.e. $\nu(X)=1$. We say that $\nu$ is non-splitting if there is no disjoint pair $C_{1}, C_{2} \in \mathcal{C}_{s}$ such that $\nu C_{1}>0$, $\nu C_{2}>0$ and $\nu C_{1}+\nu C_{2}=1$. For instance, Lebesgue measure on the unit disk, or the unit sphere (normalized) is non-splitting.

Proposition 15. Let $X$ be a q-space. Let $f$ be a q-function, and let $\nu$ be a normalized regular Borel (or topological) measure in $X$. Define $\mu$ on $\mathcal{A}_{s}$ by: $\mu C=$ $f(\nu C) ; C \in \mathcal{C}_{s}$ and $\mu U=f(1)-\mu(X \backslash U) ; U \in \mathcal{O}_{s}$. If either $\nu$ is non-splitting or $f$ is continuous, then $\mu$ is a solid set-function.

\section{Countable additivity}

Here we present a generalization to signed topological measures of the proof in [8] that topological measures are countably additive.

We recall the following lemmas, stated or implicit in [8], Section 3:

Lemma 16 (The Sierpiński Theorem). A compact, connected Hausdorff space cannot be decomposed into a countable family of disjoint, non-empty closed sets.

Lemma 17. Let $\mu$ be a signed topological measure on $X$ and suppose that $C \subset X$ is closed and 0-dimensional. Then the restriction of $\mu$ to the closed subsets of $C$ extends to a signed Borel measure on $C$.

Proof. The restriction gives a finitely additive signed measure on the algebra of relatively clopen subsets of $C$. Consider the simple continuous functions on $C$ (i.e. functions with (a finite number of) clopen level sets). They constitute an algebra 
of continuous functions which is uniformly dense in all continuous functions on $C$. (The relatively clopen subsets of $C$ are a basis for the relatively open subsets of $C$.) For a simple continuous function $f$ on $C$ define

$$
\phi(f)=\sum_{r \in \mathbb{R}} r \mu\left(f^{-1}(r)\right)=\sum_{i=1}^{n} r_{i} \mu\left(f^{-1}\left(r_{i}\right)\right),
$$

where $\left\{r_{1}, \ldots, r_{n}\right\}$ is the range of $f$. Moreover,

$$
|\phi(f)| \leq \sum_{i=1}^{n}\left|r_{i} \mu\left(f^{-1}\left(r_{i}\right)\right)\right| \leq\|f\|_{\infty}\|\mu\| .
$$

Then $\phi$ extends uniquely to a bounded linear functional on all continuous functions on $C$, and hence corresponds to a signed regular Borel measure $\nu$ in $C$. Moreover, for any relatively open set $U \subset C$ and closed set $K \subset U$ there is a relatively clopen set between them. Hence $\nu$ and $\mu$ must agree on closed subsets of $C$ by outer regularity.

Lemma 18. Let $C \subset X$ be closed, and let $Y=X / \sim$ be the quotient space obtained by identifying each component of $C$ to a point. Then $Y$ is a Hausdorff space, and the image of $C$ under the quotient map $\pi: X \rightarrow Y$ is 0-dimensional.

Theorem 19. Suppose $\left\{C_{n}: n \in \mathbb{N}\right\}$ is a disjoint collection of closed subsets of $X$ with $C=\biguplus_{n \in \mathbb{N}} C_{n}$ closed. Then $\mu(C)=\sum_{n \in \mathbb{N}} \mu\left(C_{n}\right)$.

Proof. Let $\left\{K_{i}: i \in I\right\}$ be the collection of connected components of $C$. Since $K_{i}=\biguplus_{n \in \mathbb{N}}\left(K_{i} \cap C_{n}\right)$, Lemma 16 gives that $K_{i} \subset C_{n_{i}}$ for some $n_{i}$. As in Lemma 18 let $\pi(C)$ be the image of $C$ by the quotient mapping identifying each $K_{i}$ to a point. Then $\pi^{-1}\left(\pi\left(C_{i}\right)\right)=C_{i}$ and $\pi^{-1}(\pi(C))=C$, since each $C_{n}$ is a union of components of $C$, and also $\pi(C)=\biguplus_{n \in \mathbb{N}} \pi\left(C_{n}\right)$.

By Lemma 17, the restriction of the signed topological measure $\mu \circ \pi^{-1}$ to the closed subsets of the 0-dimensional subspace $\pi(C)$ extends to a signed Borel measure on $C$, and in particular it is countably additive there. So

$$
\mu(C)=\left(\mu \circ \pi^{-1}\right)(\pi(C))=\sum_{n \in \mathbb{N}}\left(\mu \circ \pi^{-1}\right)\left(\pi\left(C_{n}\right)\right)=\sum_{n \in \mathbb{N}} \mu\left(C_{n}\right) .
$$

Corollary 20. Every signed topological measure on a compact Hausdorff space $X$ is countably additive.

Proof. As in [8, this follows from the above theorem together with the known countable additivity on open sets (following easily from inner regularity).

\section{Topological Measures as a DUAL SPACE}

In 2 a weak topology for $Q(X)$ was introduced. We extend this to $Q_{S}(X)$. The space $Q_{S}(X)$ has a vector space structure in the obvious way, i.e. $(a \mu+b \nu)(A)=$ $a \mu(A)+b \nu(A)$ for all $a, b \in \mathbb{R}, A \in \mathcal{A}(X)$ and $\mu, \nu \in Q_{S}(X)$. For each function $f \in C(X)$, let $\hat{f}$ on $Q_{S}(X)$ be given by $\hat{f}(\mu)=\mu(f)=\int x d \mu_{f}(x)$. The map $f \mapsto \hat{f}$ is a non-linear "Gelfand" transform, and $\hat{f}$ is a functional on $Q_{S}(X)$. Indeed, for 
$a \in \mathbb{R}, A \in \mathcal{A}(\mathbb{R}), \mu, \nu \in Q_{S}(X)$ and $f \in C(X)$, we have

$$
\begin{aligned}
(a \mu)_{f}(A) & =(a \mu)\left(f^{-1}(A)\right)=a\left(\mu\left(f^{-1}(A)\right)\right)=a\left(\mu_{f}(A)\right), \\
(\mu+\nu)_{f}(A) & =(\mu+\nu)\left(f^{-1}(A)\right)=\mu\left(f^{-1}(A)\right)+\nu\left(f^{-1}(A)\right)=\mu_{f}(A)+\nu_{f}(A),
\end{aligned}
$$

showing that $\hat{f}$ is a functional on $Q_{S}(X)$. Note that $\{\hat{f}\}_{f \in C(X)}$ is naturally a separating family of functionals on $Q_{S}(X)$, since $Q_{S}(X)$ is the space of quasi-linear functionals on $C(X)$.

We define the topology on $Q_{S}(X)$ to be the topology induced by the separating space of functionals $\{\hat{f}: f \in C(X)\}$. The following theorem illustrates that this topology on $Q_{S}(X)$ indeed is a weak*-topology:

Theorem 21. $Q_{S}(X)$ is a dual space, where the induced weak*-topology is the topology of pointwise convergence on $C(X)$.

Proof. For $f \in C(X)$ let $A_{f}$ denote the uniformly closed subalgebra generated by $f$ and the constant functions. Whenever $f \in A_{g}$ we let $i_{g}^{f}: A_{f} \rightarrow A_{g}$ denote the inclusion map. We may now construct the algebraic direct limit (of vector spaces)

$$
\mathfrak{X}=\lim _{\longrightarrow}\left\{A_{f}, i_{g}^{f}\right\}=\bigoplus_{f \in C(X)} A_{f} /\left\langle i_{g_{1}}^{f}(f) \oplus i_{g_{2}}^{f}(-f)\right\rangle .
$$

I.e., $\mathfrak{X}$ is the quotient of the vector space of formal finite sums of elements from distinct subalgebras $A_{f}$, and the linear span of formal sums of the form $i_{g_{1}}^{f}(f) \oplus$ $i_{g_{2}}^{f}(-f)$. We denote the latter span by $\mathfrak{I}$.

Defining $\|\cdot\|: \bigoplus_{f \in C(X)} A_{f} \rightarrow \mathbb{R}$ by $\bigoplus f_{i} \mapsto \sum\left\|f_{i}\right\|_{\infty}$ we get a quotient seminorm on $\mathfrak{X}$. The map $\Phi: Q_{S}(X) \rightarrow \mathfrak{X}^{*}$ is now given canonically by $(\Phi \mu)\left(\bigoplus f_{i}\right)=$ $\sum \mu\left(f_{i}\right)$. Note that $\Phi(\mu)$ is linear by construction, and it is independent of representatives in the equivalence classes of $\mathfrak{X}$, since

$$
(\Phi \mu)\left(i_{g_{1}}^{f}(f) \oplus i_{g_{2}}^{f}(-f)\right)=\mu(f)+\mu(-f)=0 .
$$

Furthermore, $\Phi$ is an isometry since

$$
\begin{aligned}
\|\mu\| & =\sup _{\|f\|_{\infty} \leq 1}|\mu(f)| \leq \sup _{\sum\left\|f_{i}\right\|_{\infty} \leq 1}\left|\sum \mu\left(f_{i}\right)\right| \\
& \leq \sup _{\sum\left\|f_{i}\right\|_{\infty} \leq 1} \sum\left|\mu\left(f_{i}\right)\right| \leq \sup _{\sum\left\|f_{i}\right\|_{\infty} \leq 1}\|\mu\| \sum\left\|f_{i}\right\|_{\infty}=\|\mu\|, \text { so } \\
\|(\Phi \mu)\| & =\sup _{\sum\left\|f_{i}\right\|_{\infty} \leq 1}\left|(\Phi \mu)\left(\bigoplus f_{i}\right)\right|=\sup _{\sum\left\|f_{i}\right\|_{\infty} \leq 1}\left|\sum \mu\left(f_{i}\right)\right|=\|\mu\| .
\end{aligned}
$$

Finally, $\Phi$ is onto: If $\nu \in \mathfrak{X}^{*}$, then since $\nu\left(i_{g_{1}}^{f}(f) \oplus i_{g_{2}}^{f}(-f)\right)=0$, the value of $\nu(f)$ is independent of the singly generated subalgebra $f$ is considered to be in. So we may define $\mu: C(X) \rightarrow \mathbb{R}$ by $\mu(f)=\nu(f)$ for $f \in C(X)$. It is straightforward to show that $\mu \in Q_{S}(X)$ and that $\Phi \mu=\nu$.

In summary, $\mathfrak{X}^{*}$ corresponds exactly to the quasi-linear functionals on $C(X)$. Weak*-convergence is preserved both ways since pointwise convergence on single elements is equivalent to pointwise convergence on direct sums of elements.

Remark 22. Constructing a predual space for $Q_{S}(X)$ was also done by D. Grubb (private communication). His construction was different from ours, and it is not clear what the weak*-topology will be for that construction. 


\section{Decomposition of A Signed topological MeAsure}

In the following, $\mu$ is a signed topological measure on $X$.

Definition 23. We define $\mu_{+}$as a real-valued set function, as follows:

(1) $\mu_{+}(U)=\sup \{\mu(K): K \subset U, K \in \mathcal{C}(X)\} ; U \in \mathcal{O}(X)$.

(2) $\mu_{+}(M)=\inf \left\{\mu_{+}(U): M \subset U, U \in \mathcal{O}(X)\right\} ; M \in \mathcal{A}(X)$.

We define $\mu_{-}=(-\mu)_{+}$.

We note that because expression (1) is monotonic in $U$, open sets automatically fulfill (2). Thus $\mu_{+}$is well defined.

Proposition 24. We have the following properties for $\mu_{+}$:

(1) $\mu_{+}$is non-negative and monotonic.

(2) $\mu_{+} \geq \mu$.

(3) $\mu_{+}(M)=\sup \left\{\mu_{+}(K): K \subset M, K \in \mathcal{C}(X)\right\} ; M \in \mathcal{A}(X)$. (I.e. $\mu_{+}$is inner regular as well as outer regular (equation (2) of the definition.))

(4) $\mu_{+}(U)=\sup \{\mu(O): O \subset U, O \in \mathcal{O}(X)\} ; U \in \mathcal{O}(X)$.

(5) $\mu_{+}\left(M_{1} \cup M_{2}\right)=\mu_{+}\left(M_{1}\right)+\mu_{+}\left(M_{2}\right)$, when $M_{1} \cap M_{2}=\emptyset$, and $M_{1}, M_{2}$ are both compact or both open.

(6) $\mu_{+}(M) \geq \sum_{i=1}^{n} \mu_{+}\left(M_{i}\right)$, when $\biguplus_{i=1}^{n} M_{i} \subset M$ for $M, M_{i} \in \mathcal{A}(X)$; $i=$ $1, \ldots, n ; n \in \mathbb{N}$.

(7) $\mu_{+}-\mu_{-}=\mu$.

(8) $\mu_{+}(X)+\mu_{-}(X)=\|\mu\|$.

Proof. (1) Item (2) of the definition implies that $\mu_{+}$is monotonic. Then $\mu_{+}(M) \geq$ $\mu_{+}(\emptyset)=\mu(\emptyset)=0$.

(2) If $U$ is open, $\varepsilon>0$, then by the regularity of $\mu$ we can find a closed set $K \subset U$ with $\mu(K)>\mu(U)-\varepsilon$, so that $\mu_{+}(U)>\mu(U)-\varepsilon$. Thus $\mu_{+}(U) \geq \mu(U)$ for $U$ open, and $\mu_{+}(K) \geq \mu(K)$ for compact sets follows by outer regularity of $\mu$ and $\mu_{+}$.

(3) For closed sets, (3) is obvious from monotonicity. For open sets it follows from the definition, monotonicity and (2).

(4) Given $U$ and $\varepsilon>0$, select a closed set $K \subset U$ with $\mu(K)>\mu_{+}(U)-\varepsilon / 2$. Then select an open set $O, K \subset O \subset U$ with $\mu(O)>\mu(K)-\varepsilon / 2>\mu_{+}(U)-\varepsilon$. This gives $(\leq)$ in $(4)$, while $(\geq)$ follows from monotonicity and $(2)$.

(5) If $M_{1}, M_{2}$ are disjoint open sets, then any compact within their union is the disjoint union of compacts within each, and vice versa, so that (5) follows from additivity of $\mu$. If $M_{1}, M_{2}$ are disjoint closed sets, (5) follows from additivity of $\mu_{+}$ on disjoint open sets $O_{1} \supset M_{1}, O_{2} \supset M_{2}$.

(6) Assume that (6) does not hold. By (3), we may then replace any open $M_{i}$ by a compact contained within it, without making (6) true. But if the $M_{i}$ are all compact, (6) follows from (5) and monotonicity, giving a contradiction.

(7) By regularity it suffices to show equality on open sets. Given $U$ open and $\varepsilon>0$, select a closed set $K \subset U$ such that $\mu(K)>\mu_{+}(U)-\varepsilon$. Then (e.g. by (4) applied to $\left.\mu_{-}\right) \mu_{-}(U) \geq-\mu(U \backslash K)=\mu(K)-\mu(U)>\mu_{+}(U)-\mu(U)-\varepsilon$, or $\mu_{+}(U)-\mu_{-}(U) \leq \mu(U)$. Switching $\mu_{+}$and $\mu_{-}$gives the opposite inequality.

(8) Let $\varepsilon>0$ and let $K \in \mathcal{C}(X)$ be such that $\left|\mu(K)-\mu_{+}(X)\right|<\varepsilon$. Then from (7) we get $\left|(-\mu(X \backslash K))-\mu_{-}(X)\right|<\varepsilon$. Then

$$
\|\mu\| \geq|\mu(K)|+|\mu(X \backslash K)| \geq \mu(K)-\mu(X \backslash K)>\mu_{+}(X)+\mu_{-}(X)-2 \varepsilon .
$$


In the other direction, let $\left(M_{i}\right)_{i=1}^{n}$, with $M_{i} \in \mathcal{A}(X) ; i=1, \ldots, n ; n \in \mathbb{N}$, be a disjoint family such that $\sum_{i=1}^{n}\left|\mu\left(M_{i}\right)\right|>\|\mu\|-\varepsilon$. Let $I_{+}=\left\{i: \mu\left(M_{i}\right)>0, i=\right.$ $1, \ldots, n\}$ and $I_{-}=\left\{i: \mu\left(M_{i}\right)<0, i=1, \ldots, n\right\}$. Then

$$
\mu_{+}(X)+\mu_{-}(X) \geq \sum_{i \in I_{+}} \mu\left(M_{i}\right)+\sum_{i \in I_{-}}\left(-\mu\left(M_{i}\right)\right)=\sum_{i=1}^{n}\left|\mu\left(M_{i}\right)\right|>\|\mu\|-\varepsilon .
$$

We have seen that $\mu_{+}$and $\mu_{-}$share many of the properties of (non-negative) topological measures. In fact, from Aarnes's axiomatization the only axiom missing so far is $\mu_{+}(K)+\mu_{+}(X \backslash K)=\mu_{+}(X)$. As the following example shows, there is no hope of getting this in general.

Example 25. Let $p_{1}, p_{2}, p_{3}, p_{4}$ be four distinct points in the sphere $S^{2}$. Define four solid set-functions as follows: For $M$ a solid set (open or closed)

$$
\begin{aligned}
& \nu_{1}(M)= \begin{cases}1, & \text { card }\left(M \cap\left\{p_{1}, p_{2}, p_{4}\right\}\right) \geq 2, \\
0, & \text { otherwise, }\end{cases} \\
& \nu_{2}(M)= \begin{cases}1, & \text { card }\left(M \cap\left\{p_{1}, p_{3}, p_{4}\right\}\right) \geq 2, \\
0, & \text { otherwise, }\end{cases} \\
& \nu_{3}(M)= \begin{cases}1, & p_{1} \in M \text { and } M \cap\left\{p_{2}, p_{3}, p_{4}\right\} \neq \emptyset, \\
0, & \text { ot }\left\{p_{2}, p_{3}, p_{4}\right\} \subset M,\end{cases} \\
& \nu_{4}(M)= \begin{cases}1, & p_{4} \in M \text { and } M \cap\left\{p_{1}, p_{2}, p_{3}\right\} \neq \emptyset, \\
0, & \text { or }\left\{p_{1}, p_{2}, p_{3}\right\} \subset M,\end{cases}
\end{aligned}
$$

It is readily verified that each of the above extends to a $\{0,1\}$-valued topological measure, and that $\nu_{1}+\nu_{2}=\nu_{3}+\nu_{4}$. Then $\nu=\nu_{1}-\nu_{3}=\nu_{4}-\nu_{2}$ is a signed topological measure, and furthermore we must have $\nu_{+} \leq \nu_{1}, \nu_{4}$.

It follows that if $\nu_{+}$were a topological measure, it would be proportional to both of the extreme topological measures $\nu_{1}$ and $\nu_{4}$, and thus identically zero. Let $M$ be a solid set containing $p_{2}$ and $p_{4}$ but not $p_{1}$ or $p_{3}$; then $\nu_{+}(M) \geq \nu(M)=1$. So $\nu_{+}$cannot be a topological measure.

We will therefore define a suitable class of set functions containing $\mu_{+}$and $\mu_{-}$.

Definition 26. If $\nu: \mathcal{A}(X) \rightarrow \mathbb{R}^{+}$is regular and monotonic, and if it satisfies

$$
\nu\left(C_{1} \uplus C_{2}\right)=\nu\left(C_{1}\right)+\nu\left(C_{2}\right)
$$

whenever $C_{1}, C_{2} \in \mathcal{C}(X)$ are disjoint, we shall call it a deficient topological measure.

We note that a deficient topological measure fulfills those parts of Proposition 24 which are phrased solely in terms of $\mu_{+}$; i.e. (1), (3), (5) and (6).

\section{Continuity And DeCOMPosition of QUASI-LINEAR FUnCTIONALS}

We now wish to define how to integrate continuous functions with respect to deficient topological measures. As in the case of topological measures, our first step is to use the function to move the deficient topological measure to the real line. 
Proposition 27. If $\nu: \mathcal{A}(X) \rightarrow \mathbb{R}^{+}$is a deficient topological measure and $f: X \rightarrow$ $Y$ is continuous, then $\nu \circ f^{-1}: \mathcal{A}(Y) \rightarrow \mathbb{R}^{+}$is a deficient topological measure.

Proof. The monotonicity and additivity requirements are obviously fulfilled for $\nu \circ$ $f^{-1}$. We need to show regularity. Let $\varepsilon>0$ and $O \in \mathcal{O}(Y)$ be arbitrary, and select $C \subset f^{-1}(O), C \in \mathcal{C}(X)$ (by the inner regularity of $\nu$ ) with $\nu(C)>\left(\nu \circ f^{-1}\right)(O)-\varepsilon$. Then $f(C) \subset O$ with $f(C) \in \mathcal{C}(Y)$, and $\left(\nu \circ f^{-1}\right)(f(C)) \geq \nu(C)>\left(\nu \circ f^{-1}\right)(O)-\varepsilon$, establishing the inner regularity of $\nu \circ f^{-1}$.

Now let $\varepsilon>0$ and $C \in \mathcal{C}(Y)$ be arbitrary and select $O \supset f^{-1}(C), O \in \mathcal{O}(X)$ with $\nu(O)<\left(\nu \circ f^{-1}\right)(C)+\varepsilon$. Then $Y \backslash f(X \backslash O) \in \mathcal{O}(Y)$ and $Y \backslash f(X \backslash O) \supset C$, moreover $f^{-1}(C) \subset f^{-1}(Y \backslash f(X \backslash O)) \subset O$, so $\left(\nu \circ f^{-1}\right)(Y \backslash f(X \backslash O))<\left(\nu \circ f^{-1}\right)(C)+\varepsilon$ establishing the outer regularity of $\nu \circ f^{-1}$.

Now consider a deficient topological measure $\nu$ and an $f \in C(X)$. Unlike in the case of topological measures, $\nu \circ f^{-1}$ cannot itself be extended to a Borel measure, as it is not additive on complements. However, since it is monotonic and regular, its restriction to leftward infinite closed intervals is extensible to a Borel measure, which moreover still has support in the compact set $f(X)$. In particular, the function $g(x)=\left(\nu \circ f^{-1}\right)((-\infty, x])$ is continuous from the right.

Definition 28. Given a deficient topological measure $\nu$ and $f \in C(X, \mathbb{R})$, let $f(\nu)$ be the Borel measure defined by extension of

$$
f(\nu)((-\infty, x])=\left(\nu \circ f^{-1}\right)((-\infty, x]) ; x \in \mathbb{R}
$$

(equivalently, $x \mapsto\left(\nu \circ f^{-1}\right)((-\infty, x])$ is the cumulative distribution of $\left.f(\nu)\right)$. We then define the integral of $f$ with respect to $\nu$ as

$$
\nu(f)=\int_{-\infty}^{\infty} x d f(\nu)(x)
$$

We note that even such a simple change as using rightward infinite intervals will in general give a different integral. We also note that if we use this construction with a (possibly signed) topological measure $\mu$ instead, then $f(\mu)$ becomes equal to $\mu_{f}$, and so the integral is the usual quasi-integral.

Proposition 29. We have the following properties of the integral:

(1) $\mu_{+}(a f+b)=a \mu_{+}(f)+b \mu_{+}(X) ; a \geq 0, b \in \mathbb{R}$.

(2) $f \leq g$ implies $\mu_{+}(f) \leq \mu_{+}(g)$.

(3) $\mu_{+}(f)-\mu_{-}(f)=\mu(f)$.

Proof. (1) For $a>0, x \in \mathbb{R}$ we have

$$
(a f+b)\left(\mu_{+}\right)((-\infty, a x+b])=f\left(\mu_{+}\right)((-\infty, x])
$$

so that

$$
\begin{aligned}
\mu_{+}(a f+b) & =\int_{-\infty}^{\infty} x d(a f+b)\left(\mu_{+}\right)(x)=\int_{-\infty}^{\infty}(a x+b) d f\left(\mu_{+}\right)(x) \\
& =a \int_{-\infty}^{\infty} x d f\left(\mu_{+}\right)(x)+b \lim _{x \rightarrow \infty} f\left(\mu_{+}\right)((-\infty, x]) \\
& =a \mu_{+}(f)+b \mu_{+}(X) .
\end{aligned}
$$

For $a=0, f$ does not affect the value of the integral, so we may assume $f=0$; then $a$ does not affect the value, and we may reduce to the $a>0$ case. 
(2) If $f \leq g$, then for each $x \in \mathbb{R}$ we have $f^{-1}((-\infty, x]) \supset g^{-1}((-\infty, x])$ and so $f\left(\mu_{+}\right)((-\infty, x]) \geq g\left(\mu_{+}\right)((-\infty, x])$.

Let $M>0$ be such that $\|f\|_{\infty},\|g\|_{\infty}<M$, so that both $f\left(\mu_{+}\right)$and $g\left(\mu_{+}\right)$have support in $(-M, M)$. Since the identity $x \mapsto x$ on $[-M, M]$ is continuous, and both it and $x \mapsto f\left(\mu_{+}\right)((-\infty, x])$ are of bounded variation there, we may integrate by parts:

$$
\begin{aligned}
\mu(f) & =\int_{-M}^{M} x d f\left(\mu_{+}\right)(x) \\
& =\left.\left(x f\left(\mu_{+}\right)((-\infty, x])\right)\right|_{x=-M} ^{M}-\int_{-M}^{M} f\left(\mu_{+}\right)((-\infty, x]) d x \\
& =M \mu_{+}(X)-\int_{-M}^{M} f\left(\mu_{+}\right)((-\infty, x]) d x \\
& \leq M \mu_{+}(X)-\int_{-M}^{M} g\left(\mu_{+}\right)((-\infty, x]) d x \\
& =\mu(g) .
\end{aligned}
$$

(3) In addition to Proposition 24(7), we need merely note that for a fixed $f$, Definition 28 is linear in the deficient topological measure.

Theorem 30. Every quasi-linear functional $\mu: C(X, \mathbb{R}) \rightarrow \mathbb{R}$ is uniformly continuous; specifically, for $f, g \in C(X, \mathbb{R})$

$$
|\mu(f)-\mu(g)| \leq\|\mu\|\|f-g\|_{\infty} .
$$

Proof. We have $f-\|f-g\|_{\infty} \leq g \leq f+\|f-g\|_{\infty}$, so by Proposition 29]

$$
\begin{aligned}
& \mu_{+}(f)-\mu_{+}(X)\|f-g\|_{\infty} \leq \mu_{+}(g) \leq \mu_{+}(f)+\mu_{+}(X)\|f-g\|_{\infty}, \\
& \mu_{-}(f)-\mu_{-}(X)\|f-g\|_{\infty} \leq \mu_{-}(g) \leq \mu_{-}(f)+\mu_{-}(X)\|f-g\|_{\infty},
\end{aligned}
$$

and then by Proposition 24(8)

$$
\begin{aligned}
\mu(f)-\|\mu\|\|f-g\|_{\infty} & =\mu(f)-\left(\mu_{+}(X)+\mu_{-}(X)\right)\|f-g\|_{\infty} \\
& \leq \mu(g) \\
& \leq \mu(f)+\left(\mu_{+}(X)+\mu_{-}(X)\right)\|f-g\|_{\infty} \\
& =\mu(f)+\|\mu\|\|f-g\|_{\infty},
\end{aligned}
$$

giving the result.

Remark 31. The above shows that in the definition of a quasi-linear functional, it is equivalent to assume continuity at zero, continuity everywhere, or uniform continuity.

\section{Decomposition into topological Measures}

We would like to investigate when it is possible to write a signed topological measure as the difference of two positive ones. As Example 25 showed, such a decomposition need not be unique even when it exists. Note that the decompositions $\nu_{1}-\nu_{3}$ and $\nu_{4}-\nu_{2}$ of that example are both minimal in the sense that neither of the pairs $\left(\nu_{1}, \nu_{3}\right)$ and $\left(\nu_{2}, \nu_{4}\right)$ can bound any common positive topological measure other than zero, by an argument similar to the one showing $\nu_{+}$is not a topological measure. 
From its definition and properties, we may characterize $\mu_{+}$as follows: $\mu_{+}$is the unique smallest positive monotone regular function bounding $\mu$ from above. Clearly if $\mu=\mu_{1}-\mu_{2}$, where $\mu_{1}, \mu_{2}$ are positive topological measures, then $\mu_{1}$ must have the same properties. Thus we have the following proposition:

Proposition 32. A positive topological measure $\mu^{\prime}$ gives a decomposition of a signed topological measure $\mu$ into a difference of positive topological measures by $\mu=\mu^{\prime}-\left(\mu^{\prime}-\mu\right)$, if and only if $\mu^{\prime} \geq \mu_{+}$.

In the following, let $X$ be a q-space. We may then use the full power of the solid set-function construction to construct a positive topological measure bounding $\mu_{+}$from above, and thus giving a decomposition of $\mu$. Our proof will use a few elementary results from Section 8 , which do not depend on this one.

Definition 33. Let $\nu$ be a deficient topological measure, and let $p \in X$ be an arbitrary point. We then define $\nu^{p}: \mathcal{A}_{s}(X) \rightarrow \mathbb{R}^{+}$by

$$
\nu^{p}(M)= \begin{cases}\nu(M), & p \notin M \\ \nu(X)-\nu(X \backslash M), & p \in M\end{cases}
$$

Proposition 34. We have the following properties for $\nu^{p}$ :

(1) $\nu^{p}(X)=\nu(X)$.

(2) $\nu^{p}$ is a solid set-function, and so extends to a (positive) topological measure on $X$.

(3) $\nu^{p}(M) \geq \nu(M) ; M \in \mathcal{A}(X)$.

Proof. (1) Obvious.

(2) Since $\nu(X)-\nu(X \backslash M) \geq \nu(M)$ for any set in $\mathcal{A}(X)$, and since a set containing $p$ cannot be a subset of a set not containing $p, \nu^{p}$ is monotonic on solid sets.

If $U$ is an open solid set, $p \in U$, then we may add $p$ to any compact set approximating $U$ from within, and then find a solid compact subset of $U$ containing the resulting compact set. If $p \notin U$, then any compact set contained in $U$ cannot contain $p$. Thus inner regularity of $\nu^{p}$ follows from the regularity of each defining expression.

Additivity on trivial partitions is built into the definition.

Let $M_{i} ; i=1, \ldots, n ; n \in \mathbb{N}$, be disjoint solid sets, $M \supset \biguplus_{i=1}^{n} M_{i}$, with $M$ a solid set. If none of the $M_{i}$ contains $p$, then

$$
\nu^{p}(M) \geq \nu(M) \geq \sum_{i=1}^{n} \nu\left(M_{i}\right)=\sum_{i=1}^{n} \nu^{p}\left(M_{i}\right) .
$$

If $p \in M_{1}$, say, then by the above

$$
\begin{aligned}
\nu^{p}\left(X \backslash M_{1}\right) & \geq \sum_{i=2}^{n} \nu^{p}\left(M_{i}\right)+\nu^{p}(X \backslash M) \\
\text { i.e. } & \\
\nu^{p}(M) & =\nu^{p}(X)-\nu^{p}(X \backslash M) \\
& \geq \nu^{p}(X)-\nu^{p}\left(X \backslash M_{1}\right)+\sum_{i=2}^{n} \nu^{p}\left(M_{i}\right)=\sum_{i=1}^{n} \nu^{p}\left(M_{i}\right) .
\end{aligned}
$$

Thus $\nu^{p}$ is a solid set-function. 
(3) Let $M$ be a connected compact set, and let $\left(M_{i}\right)_{i \in I}$ be the family of (open solid) components of its complement. If $p \in M$, then

$$
\begin{aligned}
\nu^{p}(M) & =\nu^{p}(X)-\sum_{i \in I} \nu^{p}\left(M_{i}\right) \\
& =\nu(X)-\sum_{i \in I} \nu\left(M_{i}\right) \\
& \geq \nu(M) .
\end{aligned}
$$

If $p \in M_{1}$, say, then

$$
\begin{aligned}
\nu^{p}(M) & =\nu^{p}(X)-\sum_{i \in I} \nu^{p}\left(M_{i}\right) \\
& =\nu(X)-\left(\nu(X)-\nu\left(X \backslash M_{1}\right)\right)-\sum_{i \in I \backslash\{1\}} \nu\left(M_{i}\right) \\
& =\nu\left(X \backslash M_{1}\right)-\sum_{i \in I \backslash\{1\}} \nu\left(M_{i}\right) \\
& \geq \nu(M) .
\end{aligned}
$$

Let $U$ be an arbitrary open set. Then by Proposition 41 and inner regularity $\nu^{p}(U)$ and $\nu(U)$ may be approximated by the values of $\nu^{p}$ and $\nu$ on finite disjoint unions of compact connected sets contained in $U$, and so satisfy the inequality. Arbitrary compact sets then follow by outer regularity.

Theorem 35. Any signed topological measure $\mu$ on a q-space $X$ may be written as the difference of positive topological measures $\mu_{1}$ and $\mu_{2}$. Moreover, we may require $\|\mu\|=\mu_{1}(X)+\mu_{2}(X)$.

Proof. Define $\mu_{1}=\mu_{+}^{p}$ and $\mu_{2}=\mu_{-}^{p}$. Then for any solid set $M$ not containing $p$, $\mu(M)=\mu_{1}(M)-\mu_{2}(M)$ and $\mu(X)=\mu_{+}(X)-\mu_{-}(X)=\mu_{1}(X)-\mu_{2}(X)$, which by taking complements gives $\mu(M)=\mu_{1}(M)-\mu_{2}(M)$ for solid sets $M$ containing $p$. By uniqueness of extension of signed solid set-functions to signed topological measures (Proposition 42), $\mu=\mu_{1}-\mu_{2}$. Finally, $\|\mu\|=\mu_{+}(X)+\mu_{-}(X)=\mu_{1}(X)+\mu_{2}(X)$.

\section{Signed SOLID SET-FUnCTIONS}

We wish to extend Aarnes's construction of a topological measure from its values at solid sets to signed topological measures. The extension definition is quite similar, replacing suprema by more general limits of nets. However, checking that a "signed solid set-function" corresponds to a signed topological measure presents additional difficulties, since the lack of monotonicity means that boundedness of the measure on solid sets, or even connected sets, is no longer sufficient to check boundedness of the signed topological measure. Also, limits need special care since sets "squeezed" between sets close in measure need no longer themselves be close in measure.

8.1. Topological preliminaries. For the entirety of this section, $X$ will be a connected, locally connected, compact Hausdorff space.

We start with a subsection of topological results, probably well known. First we have a generalization of Proposition $7(2)$. 
Proposition 36. Let $M$ be a subset of $X$, and let $C$ be a component of $M$. Then each co-component of $C$ contains a co-component of $M$. In particular, if $M$ has a finite number of co-components, then so does $C$, and if $M$ is co-connected, $C$ is solid if $C \in \mathcal{A}$.

Proof. Let $D$ be a co-component of $C$, and assume that it contains no co-component of $M$. Since any co-component of $M$ is a connected set contained in $X \backslash M \subset X \backslash C$, $D$ cannot intersect it without containing it, and so $D \subset M$. But then $D$ must be contained in a component of $M$ other than $C$, and similarly that component must be contained in $D$. So $D$ is a component of $M$.

Since $C$ and $D$ are distinct components of $M$, the closure of each does not intersect the other. But then the closure of $D$ is a connected set disjoint from $C$ and containing $D$. Since $D$ is a co-component of $C$ it must therefore be its own closure.

But also $D \subset X \backslash \bar{C} \subset X \backslash C$, so $D$ is a closed component of the open set $X \backslash \bar{C}$, a contradiction since $X$ is locally connected.

Definition 37. By $\mathcal{C}_{0}$ we denote the family of closed sets with a finite number of components. By $\mathcal{C}_{f}$ we denote the subfamily of $\mathcal{C}_{0}$ consisting of those sets whose components each have a finite number of co-components. By $\mathcal{O}_{0}$ we denote the family of open sets whose complements are in $\mathcal{C}_{0}$. We also put $\mathcal{A}_{0}=\mathcal{C}_{0} \cup \mathcal{O}_{0}$.

Corollary 38. Each (open) component of an $\mathcal{O}_{0}$-set has a finite number of cocomponents, each a compact solid set.

Proof. In Proposition [36, first consider $M$ to be the open set in question, and secondly consider $M$ to be the complement of any of its components.

Notation 39. For each $A \in \mathcal{A}_{0}$, we write its components with indices, i.e. $A_{1}, A_{2}$, $\ldots, A_{i}, \ldots$ Likewise we write each (solid) co-component of $A_{i}$ as $A_{i}^{j}$. Thus $A=$ $\biguplus_{i}\left(X \backslash \biguplus_{j} A_{i}^{j}\right)$. This notation is unique up to obvious permutations.

Lemma 40. Let $C \in \mathcal{C}_{c}$ with $X \backslash C=\biguplus_{i \in I} C^{i}$, where $\left\{C^{i}\right\}_{i \in I} \subset \mathcal{O}_{s}$ is a disjoint collection. Then $X \backslash\left(\biguplus_{i \in I_{0}} C^{i}\right) \in \mathcal{C}_{c}$ for any set of indices $I_{0} \subset I$.

Proof. Obviously $C^{\prime}=X \backslash\left(\biguplus_{i \in I_{0}} C^{i}\right) \in \mathcal{C}$. Suppose $C^{\prime}$ is not connected. Then there must be disjoint open sets $U$ and $V$ such that $\emptyset \neq C^{\prime} \cap U, C^{\prime} \cap V \in \mathcal{C}$, but $C$ and $\left\{C^{i}\right\}$ are all connected, hence they are each contained in either $U$ or $V$ respectively. We may assume without loss of generality that $C \subset U$, then $C^{\prime} \cap V=\biguplus_{C^{i} \subset C^{\prime} \cap V} C^{i} \in \mathcal{O}$. Now we reach a contradiction, since $X$ is connected and therefore contains no non-trivial clopen sets.

Proposition 41. Let $C \in \mathcal{C}, U \in \mathcal{O}, C \subset U$. Then there exists $C^{\prime} \in \mathcal{C}_{f}$ such that $C \subset C^{\prime} \subset U$.

Proof. Only a finite number of components of $U$ may intersect $C$, so it suffices to consider the case of $U$ connected. First by Proposition 7(1) let $C \subset C^{\prime \prime} \subset U$ with $C^{\prime \prime}$ connected. Now we note that a finite number of components of $X \backslash C^{\prime \prime}$ cover $X \backslash U$; we obtain by Lemma $40 C^{\prime}$ as the complement of their union. 
8.2. Definition of signed solid set-functions. The following proposition describes our general plan for recovering the value of a signed topological measure from its values at solid sets.

Proposition 42. Let $\mu$ be a signed topological measure. Then:

(1) $\mu(C)=\sum_{i}\left(\mu(X)-\sum_{j} \mu\left(C_{i}^{j}\right)\right) ; C \in \mathcal{C}_{0}$.

(2) $\mu(U)=\lim _{C \in \mathcal{C}_{0}} \mu(C) ; U \in \mathcal{O}$.

(3) $\mu(C)=\mu(X)-\mu(X \backslash C) ; C \in \mathcal{C}$.

Proof. Items (1) and (3) are obvious from additivity of $\mu$. By Proposition 41, it suffices to consider $\mathcal{C}_{0}$-sets (or even $\mathcal{C}_{f}$-sets) in the limit of $(2)$.

Definition 43. A function $\mu: \mathcal{A}_{s} \rightarrow \mathbb{R}$ is a signed solid set-function if it satisfies:

(1) There exists $M \geq 0$ such that for all $C \in \mathcal{C}_{f}$,

$$
\left|\sum_{i}\left(\mu(X)-\sum_{j} \mu\left(C_{i}^{j}\right)\right)\right| \leq M
$$

(2) For all $U \in \mathcal{O}_{s}$ we have

$$
\mu(U)=\lim _{\substack{C \subset U \\ C \in \mathcal{C}_{s}}} \mu(C)
$$

(3) For any trivial or irreducible partition $\left\{A_{i}\right\}_{i \in I}$ of $X$ we have

$$
\mu(X)=\sum_{i \in I} \mu\left(A_{i}\right)
$$

(4) $\mu(\emptyset)=0$.

Proposition 44. Let $\mu$ be a signed solid set-function. Then $\mu$ may be extended to $\mathcal{A}_{0}$ by:

(1) $\mu(C)=\sum_{i}\left(\mu(X)-\sum_{j} \mu\left(C_{i}^{j}\right)\right) ; C \in \mathcal{C}_{0}$.

(2) $\mu(U)=\mu(X)-\mu(X \backslash U) ; U \in \mathcal{O}_{0}$.

In particular, the extension of $\mu$ is bounded by $M$ on $\mathcal{C}_{0}$ and by $2 M$ on $\mathcal{O}_{0}$.

Proof. If $C \in \mathcal{C}_{0}$, then for any component $C_{i}$ of $C,\left|\sum_{j \in J^{\prime}} \mu C_{i}^{j}\right| \leq M+|\mu(X)|$ for any finite index subset $J^{\prime}$, by Definition 43(1) and Lemma 40. In particular, the sum $\sum_{j} \mu\left(C_{i}^{j}\right)$ is absolutely convergent. Hence $\mu$ is well defined and real-valued on $\mathcal{A}_{0}$. Given $\varepsilon>0$, let $\left\{C_{i}\right\}_{i=1}^{n}$ be the components of $C$, and for each $i$ pick $n_{i}$ such that

$$
\left|\mu\left(C_{i}\right)-\left(\mu(X)-\sum_{j=1}^{n_{i}} \mu\left(C_{i}^{j}\right)\right)\right|<\frac{\varepsilon}{n}
$$

and such that $C_{k} \subset \biguplus_{j=1}^{n_{i}} C_{i}^{j}$ for $k \neq i$. This gives $C_{k} \subset X \backslash \biguplus_{j=1}^{n_{k}} C_{k}^{j} \subset X \backslash C_{i}$, so the connected set $X \backslash \biguplus_{j=1}^{n_{k}} C_{k}^{j}$ must be in the same co-component of $C_{i}$ as $C_{k}$ is, and thus $X \backslash \biguplus_{j=1}^{n_{k}} C_{k}^{j} \subset \biguplus_{j=1}^{n_{i}} C_{i}^{j}$. Then the set $C^{\prime}=\biguplus_{i=1}^{n}\left[X \backslash\left(\biguplus_{j=1}^{n_{i}} C_{i}^{j}\right)\right]$ is in $\mathcal{C}_{f}$ and we get

$$
\left|\mu(C)-\mu\left(C^{\prime}\right)\right| \leq \sum_{i=1}^{n}\left|\mu\left(C_{i}\right)-\left(\mu(X)-\sum_{j=1}^{n_{i}} \mu\left(C_{i}^{j}\right)\right)\right|<\varepsilon .
$$


Accordingly $\mu$ is bounded by $M$ on $\mathcal{C}_{0}$. By (2), $\mu$ must be bounded by $2 M$ on $\mathcal{A}_{0}$. One may verify that the extension is consistent with the definition of $\mu$ on $\mathcal{A}_{s}$.

Remark 45. We will assume from now on that $\mu$ is a signed solid set-function extended to $\mathcal{A}_{0}$ according to the proposition above.

Corollary 46. We have the following properties for $\mu$ :

(1) Let $C, C^{\prime} \in \mathcal{C}_{0}$ with $C \cap C^{\prime}=\emptyset$; then $\mu\left(C \uplus C^{\prime}\right)=\mu(C)+\mu\left(C^{\prime}\right)$.

(2) Let $C \in \mathcal{C}_{0}, U \in \mathcal{O}_{0}, C \subset U$; then $\mu(U \backslash C)=\mu(U)-\mu(C)$.

(3) Let $C \in \mathcal{C}_{c}$ with co-components $\left\{C^{j}\right\}_{j \in J}$. Then for any subset $I \subset J$ we have

$$
\mu\left(\biguplus_{j \in I} C^{j}\right)=\sum_{j \in I} \mu\left(C^{j}\right) .
$$

(4) Let $\left(C_{i}\right)_{i \in I} \subset \mathcal{C}_{0}$ be a disjoint family of $\mathcal{C}_{0}$-sets; then $\sum_{i}\left|\mu\left(C_{i}\right)\right| \leq 2 M$.

(5) Let $\left(U_{i}\right)_{i \in I} \subset \mathcal{O}_{s}$ be a disjoint family of solid open sets; then $\sum_{i}\left|\mu\left(U_{i}\right)\right| \leq$ $2 M$.

Proof. (1) The family $\mathcal{C}_{0}$ is closed under finite unions, and the components of a disjoint union are the components of the individual sets.

(2) This follows from (1) by considering $C^{\prime}=X \backslash U$.

(3) $C^{\prime}=X \backslash\left(\biguplus_{j \in I} C^{j}\right) \in \mathcal{C}_{c}$ by Lemma 40 and hence

$$
\begin{aligned}
\mu\left(\biguplus_{j \in I} C^{j}\right) & =\mu(X)-\mu\left(C^{\prime}\right)=\mu(X)-\left(\mu(X)-\sum_{j \in I} \mu\left(C^{j}\right)\right) \\
& =\sum_{j \in I} \mu\left(C^{j}\right) .
\end{aligned}
$$

(4) It suffices to consider finite families. Let $I_{+}=\left\{i: \mu\left(C_{i}\right)>0\right\}$ and $I_{-}=I \backslash I_{+}$. Then

$$
\sum_{i}\left|\mu\left(C_{i}\right)\right|=\sum_{i \in I_{+}}\left|\mu\left(C_{i}\right)\right|+\sum_{i \in I_{-}}\left|\mu\left(C_{i}\right)\right|=\left|\mu\left(\biguplus_{i \in I_{+}} C_{i}\right)\right|+\left|\mu\left(\biguplus_{i \in I_{-}} C_{i}\right)\right| \leq 2 M .
$$

(5) Let $\varepsilon>0$. By Definition 43(2) we may find $C_{i} \in \mathcal{C}_{s}, C_{i} \subset U_{i}$ with $\left|\mu\left(C_{i}\right)\right| \geq$ $(1-\varepsilon)\left|\mu\left(U_{i}\right)\right|$, from which (4) gives the result.

8.3. Regularity on $\mathcal{A}_{0}$. We will now embark on proving the result (Proposition 51) that the signed solid set-functions are regular on $\mathcal{A}_{0}$. For positive topological measures, this essentially corresponds to condition $(\mathrm{Q} 3)_{0}$ of [3], Section 4. Condition $(\mathrm{Q} 1)_{0}$ is monotonicity, which we do not have; condition $(\mathrm{Q} 2)_{0}$ is additivity on disjoint $\mathcal{C}_{0}$-sets, which is Corollary 46)(1).

Lemma 47. Given $\varepsilon>0$ and $C \in \mathcal{C}_{s}$, there is a $U \in \mathcal{O}_{s}, C \subset U$ such that $K \in \mathcal{C}_{0}, K \subset U \backslash C$ implies $|\mu(K)|<\varepsilon$.

Proof. Assume to the contrary that no such set $U \in \mathcal{O}_{s}$ exists. Then recursively there is a set $K_{i} \in \mathcal{C}_{0}, K_{i} \subset O_{i} \backslash C$ with $\left|\mu\left(K_{i}\right)\right| \geq \varepsilon$ and by Proposition 7(3) a set $O_{i+1} \in \mathcal{O}_{s}$ with $C \subset O_{i+1} \subset O_{i} \backslash K_{i}\left(O_{1}\right.$ may be taken to be $\left.X\right)$. Now for each $n$ we have $\sum_{i=1}^{n}\left|\mu\left(K_{i}\right)\right| \geq \varepsilon n$, eventually violating Corollary 46(4).

Lemma 48. If $U \in \mathcal{O}_{c}$ has a finite number of co-components, which are all solid, then

$$
\mu(U)=\lim _{\substack{C \subset U \\ C \in \mathcal{C}_{0}}} \mu(C)
$$


Proof. Let $\left\{K_{i}\right\}_{i=1}^{n}$ denote the co-components of $U$. Given $\varepsilon>0$ pick sets $\left\{U_{i}\right\}_{i=1}^{n} \subset$ $\mathcal{O}_{s}$ such that $K \in \mathcal{C}_{0}$ with $K \subset U_{i} \backslash K_{i}$ implies $|\mu(K)|<\frac{\varepsilon}{n}$ and such that $U \in \mathcal{O}_{s}$ with $K_{i} \subset U \subset U_{i}$ implies $\left|\mu(U)-\mu\left(K_{i}\right)\right|<\frac{\varepsilon}{n}$ (by Lemma47 and Definition 433(2), respectively). By Proposition $7(1)$ there is a set $C \in \mathcal{C}_{c}$ with $X \backslash\left(\bigcup_{i \in I} U_{i}\right) \subset C \subset U$. By Lemma 40 we may assume that all co-components $C^{j}$ contain exactly one $K_{i}$ (replace $C$ with $\left.C \cup\left(\bigcup\left\{C^{j}: C^{j} \cap\left(\bigcup_{i=1}^{n} K_{i}\right)=\emptyset\right\}\right)\right)$. We now claim that $F \in \mathcal{C}_{0}, C \subset F \subset U$ implies $|\mu(U)-\mu(F)|<5 \varepsilon$ (completing the proof).

One of the components, say $F^{\prime}$, of $F$ must contain $C$ (since $C$ is connected) and $F \backslash F^{\prime} \in \mathcal{C}_{0}$ with $F \backslash F^{\prime} \subset \bigcup_{i=1}^{n} U_{i} \backslash K_{i}, F \backslash F^{\prime} \cap U_{i} \in \mathcal{C}_{0}$ for $i=1, \ldots, n$. Hence $\left|\mu\left(F \backslash F^{\prime}\right)\right|<\varepsilon$.

Let $\left\{V_{i}\right\}_{i=1}^{\infty}$ (possibly finite collection) be the co-components of $F^{\prime}$ where $\mu$ is non-zero and $V_{i} \cap\left(\bigcup_{i=1}^{n} K_{i}\right)=\emptyset$. Pick $F_{i} \in \mathcal{C}_{s}, F_{i} \subset V_{i}$ with $\left|\mu\left(V_{i}\right)-\mu\left(F_{i}\right)\right|<\frac{\varepsilon}{2^{2}}$ for $i=1,2, \ldots$ Now $\bigcup_{i=1}^{\infty} F_{i} \subset \bigcup_{i=1}^{n} U_{i} \backslash K_{i}$ and $\bigcup_{i=1}^{m} F_{i} \in \mathcal{C}_{0}$ for $m \in \mathbb{N}$, so $\left|\mu\left(\bigcup_{i=1}^{m} F_{i}\right)\right|<\varepsilon$. For $m$ sufficiently large we have $\left|\mu\left(\bigcup_{i=1}^{\infty} V_{i}\right)-\mu\left(\bigcup_{i=1}^{m} F_{i}\right)\right|<2 \varepsilon$, implying $\left|\mu\left(\bigcup V_{i}\right)\right|=\left|\sum \mu\left(V_{i}\right)\right|<3 \varepsilon$ (cf. Corollary 46(3)).

Finally, we have

$$
|\mu(U)-\mu(F)| \leq\left|\mu(U)-\mu\left(F^{\prime}\right)\right|+\varepsilon \leq\left|\mu(U)-\mu\left(F^{\prime} \cup\left(\bigcup V_{i}\right)\right)\right|+4 \varepsilon<5 \varepsilon,
$$

where the last inequality is due to each co-component of $F^{\prime} \cup\left(\bigcup V_{i}\right)$ being an open solid set $U$ with $K_{i} \subset U \subset U_{i}$ for some choice of $i$. The proof is complete.

Lemma 49. For all $U \in \mathcal{O}_{0}$,

$$
\lim _{\substack{C \subset U \\ C \in \mathcal{C}_{0}}} \mu C=\sum_{i} \mu U_{i}
$$

Proof. By Corollary 46(4) and monotone convergence we have

$$
\sum_{i} \sup _{\substack{C \subset U_{i} \\ C \in \mathcal{C}_{0}}}|\mu C| \leq 2 M
$$

Given $\varepsilon>0$, we may therefore write $U=U^{\prime} \uplus U^{\prime \prime}, U^{\prime}, U^{\prime \prime} \in \mathcal{O}$, where $U^{\prime}=$ $\biguplus_{i=1}^{n} U_{i}^{\prime}$ consists of a finite number of components of $U$ and

$$
\left|\sum_{i} \mu\left(U_{i}^{\prime \prime}\right)\right| \leq \sup _{\substack{C \subset U^{\prime \prime} \\ C \in \mathcal{C}_{0}}}|\mu C| \leq \sum_{i} \sup _{\substack{C \subset U_{i}^{\prime \prime} \\ C \in \mathcal{C}_{0}}}|\mu C|<\varepsilon .
$$

By Lemma 48 we then select $C_{i} \in U_{i}^{\prime}$ such that $F \in \mathcal{C}_{0}, C_{i} \subset F \subset U_{i}^{\prime}$ implies $\left|\mu\left(U_{i}^{\prime}\right)-\mu(F)\right|<\varepsilon / n$. Let $C=\biguplus_{i=1}^{n} C_{i}$.

We then have that $F \in \mathcal{C}_{0}, C \subset F \subset U$ implies

$$
\begin{aligned}
\left|\sum_{i} \mu U_{i}-\mu(F)\right| & \leq \sum_{i=1}^{n}\left|\mu\left(U_{i}^{\prime}\right)-\mu\left(F \cap U_{i}^{\prime}\right)\right|+\left|\sum_{i} \mu\left(U_{i}^{\prime \prime}\right)-\mu\left(F \cap U^{\prime \prime}\right)\right| \\
& <\sum_{i=1}^{n} \varepsilon / n+2 \varepsilon=3 \varepsilon .
\end{aligned}
$$

Lemma 50. Let $U \in \mathcal{O}_{0}$ have solid co-components. Then

$$
\mu(U)=\sum_{i \in I} \mu\left(U_{i}\right)
$$


Proof. Let $\left\{C_{i}\right\}_{i=1}^{n}$ denote the co-components of $U$. We have the equivalent statement:

$$
\mu(X)=\sum_{i \in I} \mu\left(U_{i}\right)+\sum_{i=1}^{n} \mu\left(C_{i}\right)
$$

The proof is by induction on $n$. If $n=0$, then $U=X$ and the statement is trivial. If $n=1$, then $U \in \mathcal{O}_{s}$ and we have the trivial partition. Equality follows from the definition of solid set-functions. Assume the equation to hold up to an arbitrary $n \in \mathbb{N}$. If $U$ is connected, then equality follows from the definition of $\mu$ on $\mathcal{O}_{0}$. If $U$ is not connected, then Proposition 12 applies to the collection $\left\{C_{i}\right\}_{i=1}^{n+1}$. That is, we have a subfamily $\left\{C_{i}\right\}_{i \in J}$, card $J \geq 2$, such that $X=\left(\bigcup_{i \in J} C_{i}\right) \cup\left(\bigcup_{i \in I^{\prime}} O_{i}\right)$ is an irreducible partition of $X$ (here $O=X \backslash \bigcup_{i \in J} C_{i}$ ). By Definition 43) (3) we have

$$
\mu(X)=\sum_{i \in I^{\prime}} \mu\left(O_{i}\right)+\sum_{i \in J} \mu\left(C_{i}\right)
$$

For an arbitrary choice of $i^{\prime} \in I^{\prime}$ we have

$$
O_{i^{\prime}}=\left(\bigcup_{C_{i} \subset O_{i^{\prime}}} C_{i}\right) \cup\left(\bigcup_{U_{i}^{\prime} \subset O_{i^{\prime}}} U_{i}\right)
$$

Now the induction hypothesis applies to the collection $\left\{C_{i}: C_{i} \subset O_{i^{\prime}}\right\} \cup\left\{X \backslash O_{i^{\prime}}\right\}$ since it consists of at most $n$ solid compact sets. We have

$$
\begin{aligned}
\mu(X) & =\sum_{U_{i}^{\prime} \subset O_{i^{\prime}}} \mu\left(U_{i}\right)+\sum_{C_{i} \subset O_{i^{\prime}}} \mu\left(C_{i}\right)+\mu\left(X \backslash O_{i^{\prime}}\right) \Rightarrow \\
\mu\left(O_{i^{\prime}}\right) & =\sum_{U_{i}^{\prime} \subset O_{i^{\prime}}} \mu\left(U_{i}\right)+\sum_{C_{i} \subset O_{i^{\prime}}} \mu\left(C_{i}\right) .
\end{aligned}
$$

Finally, we obtain

$$
\mu(X)=\sum_{i \in I^{\prime}} \mu\left(O_{i}\right)+\sum_{i \in J} \mu\left(C_{i}\right)=\sum_{i \in I} \mu\left(U_{i}\right)+\sum_{i=1}^{n+1} \mu\left(C_{i}\right) .
$$

The proof is complete.

Proposition 51. For all $U \in \mathcal{O}_{0}$ we have

$$
\mu(U)=\lim _{\substack{C \subset U \\ C \in \mathcal{C}_{0}}} \mu C .
$$

Proof. We will show that $\mu(U)=\sum_{i} \mu\left(U_{i}\right)$, from which the result follows by Lemma 49. Throughout the proof let $C=X \backslash U \in \mathcal{C}_{0}$.

We will first prove a special case. Assume that for each $i$ there is a $j$ such that $C_{k} \subset C_{i}^{j}$ for all $k \neq i$. Let $O=\bigcup_{j, i}\left\{C_{i}^{j}: C_{k} \cap C_{i}^{j}=\emptyset\right.$ for all $\left.k\right\}$.

Consider the sets $K_{i}=C_{i} \cup\left(\bigcup_{\left\{j: C_{k} \cap C_{i}^{j}=\emptyset \text { for all } k\right\}} C_{i}^{j}\right)$. We have $\left\{K_{i}: i \in I\right\} \subset$ $\mathcal{C}_{s}$, so $O^{\prime}=X \backslash\left(\bigcup K_{i}\right)$ is an open set in $\mathcal{O}_{0}$ with solid co-components. By Lemma 
50, $\mu\left(O^{\prime}\right)=\sum_{i} O_{i}^{\prime}$. Then

$$
\begin{aligned}
\mu(U) & =\mu(X)-\sum_{i}\left(\mu(X)-\sum_{j} \mu\left(C_{i}^{j}\right)\right) \\
& =\mu(X)-\sum_{i}\left(\mu\left(K_{i}\right)-\sum_{C_{k} \cap C_{i}^{j}=\emptyset, \forall k} \mu\left(C_{i}^{j}\right)\right) \\
& =\mu(X)-\sum_{i} \mu\left(K_{i}\right)+\sum_{i} \mu\left(O_{i}\right) \\
& =\mu\left(O^{\prime}\right)+\sum_{i} \mu\left(O_{i}\right)=\sum_{i} \mu\left(O_{i}^{\prime}\right)+\sum_{i} \mu\left(O_{i}\right) \\
& =\sum_{i} \mu\left(U_{i}\right),
\end{aligned}
$$

completing the proof for this case.

Let $n=\operatorname{card}\left\{C_{i}: i \in I\right\}$. The remaining proof will be by induction on $n$. For $n \leq 2$ we must be in the case already proved. Assume $\mu(U)=\sum_{i} \mu\left(U_{i}\right)$ to hold for $n=1,2, \ldots, m$, and let $C \in \mathcal{C}_{0}$ with $\operatorname{card}\left\{C_{i}\right\}=m+1$.

Having proved the first case, we may now assume that there are distinct $i, k_{1}, k_{2}$ together with $j$, such that $C_{k_{1}} \subset C_{i}^{j}$ but $C_{k_{2}} \cap C_{i}^{j}=\emptyset$.

Now the induction hypothesis applies to the collection

$$
K=X \backslash O=\left(C_{i} \cup C_{i}^{j}\right) \cup\left(\bigcup_{\substack{C_{k} \cap C_{i}^{j}=\emptyset \\ k \neq i}} C_{k}\right)
$$

and to the collection

$$
K^{\prime}=X \backslash O^{\prime}=\left(X \backslash C_{i}^{j}\right) \cup\left(\bigcup_{C_{k} \subset C_{i}^{j}} C_{k}\right) .
$$

Then $K \cap K^{\prime}=C, K \cup K^{\prime}=X$ (i.e. $O \cap O^{\prime}=\emptyset$ ) and

$$
\begin{aligned}
\sum_{i} \mu\left(U_{i}\right) & =\sum_{i} \mu\left(O_{i}\right)+\sum_{i} \mu\left(O_{i}^{\prime}\right)=\mu(O)+\mu\left(O^{\prime}\right) \\
& \text { (by induction) } \\
& =\left(\mu(X)-\mu\left(C_{i} \cup C_{i}^{j}\right)-\sum_{\substack{C^{k} C_{i j}^{j}=\emptyset \\
k \neq i}} \mu\left(C_{k}\right)\right) \\
& +\left(\mu(X)-\mu\left(X \backslash C_{i}^{j}\right)-\sum_{C_{k} \subset C_{i}^{j}} \mu\left(C_{k}\right)\right) \\
& =\sum_{l \neq j} \mu\left(C_{i}^{l}\right)+\mu\left(C_{i}^{j}\right)-\sum_{k \neq i} \mu\left(C_{k}\right)=\mu(X)-\mu\left(C_{i}\right)-\sum_{k \neq i} \mu\left(C_{k}\right) \\
& =\mu(U) .
\end{aligned}
$$




\subsection{Extension to $\mathcal{A}$.}

Lemma 52. Let $\mu$ be a signed solid set-function extended to $\mathcal{A}_{0}$. For any open set $U, \lim _{C \in \mathcal{C}_{0}} \mu C$ exists.

Proof. Let $C_{1} \subset U$ and $C_{1} \in \mathcal{C}_{0}$. Then, recursively, there is a set $V_{1} \in \mathcal{O}_{0}$ with $C_{1} \subset V_{1} \subset \bar{V}_{1} \subset U$ and a set $C_{2} \in \mathcal{C}_{0}$ with $V_{1} \subset C_{2} \subset U$. We obtain an increasing sequence $\left\{C_{i}\right\}$. Now suppose to the contrary that the limit does not exist. Then there is an $\varepsilon>0$ such that $\left\{C_{i}\right\}$ may be chosen with $\left|\mu C_{i}-\mu C_{i+1}\right|>\varepsilon$ and $V_{i}$ with $\left|\mu V_{i}-\mu C_{i}\right|<\frac{\varepsilon}{2}$ for $i=1,2, \ldots$.

We get $\varepsilon<\left|\mu C_{i}-\mu C_{i+1}\right|=\left|\mu C_{i}-\mu V_{i+1}+\mu V_{i+1}-\mu C_{i+1}\right| \leq\left|\mu C_{i}-\mu V_{i+1}\right|+$ $\left|\mu V_{i+1}-\mu C_{i+1}\right| \leq\left|\mu C_{i}-\mu V_{i+1}\right|+\frac{\varepsilon}{2}$; accordingly $\left|\mu C_{i}-\mu V_{i+1}\right| \geq \frac{\varepsilon}{2}$. We have constructed a disjoint sequence $\left\{V_{2 i+1} \backslash C_{2 i}\right\} \subset \mathcal{O}_{0}$ with $\left|\mu\left(V_{2 i+1} \backslash C_{2 i}\right)\right| \geq \frac{\varepsilon}{2}, i=$ $1,2, \ldots$ Finally, pick $K_{i} \subset V_{2 i+1} \backslash C_{2 i}, K_{i} \in \mathcal{C}_{0}$ with $\left|\mu\left(V_{2 i+1} \backslash C_{2 i}\right)-\mu K_{i}\right|<\frac{\varepsilon}{4}$ implying $\left|\mu K_{i}\right| \geq \frac{\varepsilon}{4}$. We reach a contradiction with $\sum_{i}\left|\mu\left(K_{i}\right)\right|=\infty$.

Theorem 53. A signed solid set-function $\mu: \mathcal{A}_{s} \rightarrow \mathbb{R}$ extends uniquely to a signed topological measure in $X$. Conversely, the restriction of a signed topological measure to the solid sets is a signed solid set-function.

Proof. For an arbitrary set in $\mathcal{A}$ we define $\mu$ by:

(1) $\mu V=\lim _{C \in \mathcal{C}_{0}} \mu C, V \in \mathcal{O}$;

(2) $\mu C=\mu X-\mu(X \backslash C), C \in \mathcal{C}$.

Clearly this is consistent with the definition on $\mathcal{A}_{0}$. We start by proving the regularity (Definition $2(3)$ ). Let $\varepsilon>0$ and $U \in \mathcal{O}$ be arbitrary. Then by Lemma 52 there is a set $C \in \mathcal{C}_{0}, C \subset U$, such that for any set $C^{\prime} \in \mathcal{C}_{0}$ with $C \subset C^{\prime} \subset U$ we have $\left|\mu C-\mu C^{\prime}\right|<\frac{\varepsilon}{2}$. Now let $K$ be any closed set with $C \subset K \subset U$. It suffices to show that $|\mu C-\mu K|<\varepsilon$. To this end, choose a set $K^{\prime} \in \mathcal{C}_{0}$ such that $X \backslash U \subset K^{\prime} \subset X \backslash K$ and $\left|\mu(X \backslash K)-\mu K^{\prime}\right|=\left|\mu\left(X \backslash K^{\prime}\right)-\mu K\right|<\frac{\varepsilon}{2}$ (by Lemma 52). Again by Lemma 52 and Proposition 51, $\left|\mu\left(X \backslash K^{\prime}\right)-\mu C\right| \leq \frac{\varepsilon}{2}$. We have

$$
|\mu C-\mu K| \leq\left|\mu C-\mu\left(X \backslash K^{\prime}\right)\right|+\left|\mu\left(X \backslash K^{\prime}\right)-\mu K\right|<\varepsilon,
$$

which establishes Definition 2(3).

For Definition 2(2), it is clear from the definition of $\mu$ on open sets that $\sup \{|\mu U|$ :

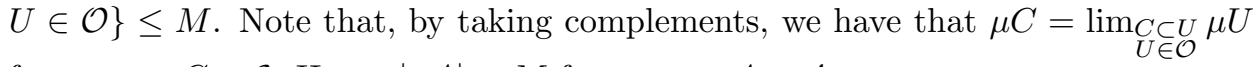
for any set $C \in \mathcal{C}$. Hence $|\mu A| \leq M$ for any set $A \in \mathcal{A}$.

It remains to prove additivity (Definition $2(1)$ ). Let $U_{1}$ and $U_{2}$ be disjoint open sets. For any pair of closed sets $C_{1}, C_{2} \in \mathcal{C}_{0}, C_{1} \subset U_{1}, C_{2} \subset U_{2}$, we have $C_{1} \cup C_{2} \in \mathcal{C}_{0}, C_{1} \cup C_{2} \subset U_{1} \cup U_{2}$. Conversely, for any $C \in \mathcal{C}_{0}, C \subset U_{1} \cup U_{2}$, we have $C \cap U_{1} \in \mathcal{C}_{0}$ and $C \cap U_{2} \in \mathcal{C}_{0}$. Hence by additivity of $\mu$ on $\mathcal{C}_{0}$ we have

$$
\mu\left(U_{1} \cup U_{2}\right)=\lim _{\substack{C \subset U_{1} \cup U_{2} \\ C \in \mathcal{C}_{0}}} \mu C=\lim _{\substack{C \subset U_{1} \\ C \in \mathcal{C}_{0}}} \mu C+\lim _{\substack{C \subset U_{2} \\ C \in \mathcal{C}_{0}}} \mu C=\mu U_{1}+\mu U_{2} .
$$

By induction $\mu$ is finitely additive on open sets. Now let $C_{1}, C_{2} \in \mathcal{C}$ be disjoint. As above we obtain

$$
\mu\left(C_{1} \cup C_{2}\right)=\lim _{\substack{C_{1} \cup C_{2} \subset U \\ U \in O}} \mu U=\lim _{\substack{C_{1} \subset U \\ U \in \mathcal{O}}} \mu U+\lim _{\substack{C_{2} \subset U \\ U \in \mathcal{O}}} \mu U=\mu C_{1}+\mu C_{2} .
$$

Finally, let $A_{1}, A_{2}, \ldots, A_{n} \in \mathcal{A}$ be a disjoint collection with $\bigcup_{i=1}^{n} A_{i} \in \mathcal{A}$. Put $A_{0}=X \backslash \bigcup_{i=1}^{n} A_{i}$; by finite additivity on open and closed sets respectively, and (2) 
we get

$$
\begin{aligned}
\mu X & =\mu\left(\bigcup_{i=0}^{n} A_{i}\right)=\mu\left(\bigcup_{A_{i} \in \mathcal{C}} A_{i}\right)+\mu\left(\bigcup_{A_{i} \in \mathcal{O}} A_{i}\right)=\mu A_{0}+\sum_{i=1}^{n} \mu A_{i} \\
& =\mu X-\mu\left(\bigcup_{i=1}^{n} A_{i}\right)+\sum_{i=1}^{n} \mu A_{i},
\end{aligned}
$$

and hence

$$
\mu\left(\bigcup_{i=1}^{n} A_{i}\right)=\sum_{i=1}^{n} \mu A_{i}
$$

The uniqueness of the extension follows from Proposition 42 .

Now assume instead $\mu$ to be a signed topological measure, and consider its restriction to $\mathcal{A}_{s}$. Definition 43(1) follows by Proposition 42(1) and Definition 2(1); Definition 43(2) follows from finite additivity, and the fact that it suffices to consider solid sets in Definition 43)(3) follows from Proposition 7(3).

\section{Signed Q-FunCtions}

The q-functions originated in [4 have proven to be an efficient tool for constructing topological measures (see e.g. [5]). It is natural to ask whether a similar construction technique might apply to signed topological measures. It turns out that this is possible.

Throughout this section we will let $X$ denote a q-space. Let $Q_{1}(X)$ denote the normalized positive topological measures (i.e. $\mu(X)=1$ ). We say that $\mu \in Q_{1}(X)$ is non-splitting if there is no disjoint pair $C_{1}, C_{2} \in \mathcal{C}_{s}$ such that $\mu\left(C_{1}\right)>0, \mu\left(C_{2}\right)>0$ and $\mu\left(C_{1}\right)+\mu\left(C_{2}\right)=1$ (Lebesgue measure on the disk or sphere are examples of non-splitting measures).

Definition 54. For $f:[0,1] \rightarrow \mathbb{R}, \mu \in Q_{1}(X), C \in \mathcal{C}_{s}$, define $f_{*}(\mu): \mathcal{C}_{s} \rightarrow \mathbb{R}$ by

$$
\left[f_{*}(\mu)\right](C)=f(\mu(C)) \text { for all } C \in \mathcal{C}_{s} .
$$

Then $f$ is called a signed $q$-function if $f_{*}(\mu)$ extends to a signed solid set-function (and hence to a signed topological measure) in $X$ for any q-space $X$, and any non-splitting $\mu \in Q_{1}(X)$.

Remark 55. If $f$ is a signed q-function, then $f_{*}(\mu)$ is determined on $\mathcal{O}_{s}$ by

$$
\left(f_{*} \mu\right)(U)=\left(f_{*} \mu\right)(X)-\left(f_{*} \mu\right)(X \backslash U)=f(1)-\left(f_{*} \mu\right)(X \backslash U) .
$$

Since $X$ is a q-space it only exhibits trivial partitions. Hence (11) assures the additivity requirement (Definition $43(3))$ of $f_{*} \mu$ trivially. For any signed q-function $f$ we must also have $f(0)=0$, since the empty set always has measure zero.

Proposition 56. If $f$ is a signed q-function, then:

(1) $f\left(x^{-}\right)+f(1-x)=f(1)$ for all $x \in(0,1]$.

(2) $f$ is continuous from the right.

(3) $f$ has bounded variation.

Proof. Let $m$ be the Lebesgue measure on the unit interval. Then $f_{*} m$ extends to a signed topological measure in $\mathbb{R}$, and hence extends uniquely to a regular signed Borel measure in $\mathbb{R}$. The proposition now follows from elementary measure theory. 
Example 57. Let $f:[0,1] \rightarrow \mathbb{R}$ be given by

$$
f(x)=\left\{\begin{array}{cl}
\sqrt{x} & , \quad x \in\left[0, \frac{1}{2}\right] \\
\sqrt{2}-\sqrt{1-x} & , \quad x \in\left[\frac{1}{2}, 1\right] .
\end{array}\right.
$$

Then $\lim _{x \rightarrow 0^{+}} f^{\prime}(x)=\infty$, so for any $M$ there is an $\varepsilon>0$ and $n \in \mathbb{N}$ such that $n \varepsilon<1$ and $n f(\varepsilon)>M$. Let $m$ be the normalized Lebesgue measure in the unit disk $D$. Choose disjoint sets $\left\{C_{i}\right\}_{i=1}^{n} \subset \mathcal{C}_{s}$ such that $m\left(C_{1}\right)=\cdots=m\left(C_{n}\right)=\varepsilon$. Assuming that $f$ is a signed q-function we have $\left(f_{*} m\right)\left(\bigcup C_{i}\right)=\sum\left(f_{*} m\right)\left(C_{i}\right)>M$, which is a contradiction. Apparently Proposition 56 does not provide sufficient conditions for a signed q-function.

In [4] it was shown that a convex function on the interval $\left[0, \frac{1}{2}\right]$ extends uniquely to a $\mathrm{q}$-function on the whole unit interval. This immediately provides us with a large class of signed q-functions:

Proposition 58. If $f:\left[0, \frac{1}{2}\right] \rightarrow \mathbb{R}$ with $f(0)=0$ is differentiable, and $f^{\prime}$ has bounded variation, then $f$ extends to a continuous signed q-function on $[0,1]$.

Proof. If $f^{\prime}$ has bounded variation, then $f^{\prime}=g-h$, where $g$ and $h$ are monotone functions. In particular, $f$ is the difference of two convex functions, say $f_{1}$ and $f_{2}$, on the interval $\left[0, \frac{1}{2}\right]$. Since $f(0)=f_{1}(0)-f_{2}(0)=0$ we may assume that $f_{1}(0)=f_{2}(0)=0$. Assuming continuity of $f$, Proposition 56 requires that $f(x)+$ $f(1-x)=f(1)(x \in[0,1])$. Accordingly we must have

$$
f(x)= \begin{cases}f_{1}(x)-f_{2}(x) & , \quad x \in\left[0, \frac{1}{2}\right] \\ f(1)-f_{1}(1-x)-f_{2}(1-x) & , \quad x \in\left[\frac{1}{2}, 1\right],\end{cases}
$$

implying that $f(1)=2 f_{1}\left(\frac{1}{2}\right)-2 f_{2}\left(\frac{1}{2}\right)$. Now $f:[0,1] \rightarrow \mathbb{R}$ is the difference of the two q-functions $k_{1}, k_{2}$ given by

$$
k_{i}(x)= \begin{cases}f_{i}(x) & , \quad x \in\left[0, \frac{1}{2}\right], \quad i=1,2 . \\ 2 f_{i}\left(\frac{1}{2}\right)-f_{i}(1-x) & , \quad x \in\left[\frac{1}{2}, 1\right],\end{cases}
$$

Example 59. Let $f(x)=2 x^{3}-3 x^{2}+2 x:[0,1] \rightarrow \mathbb{R}$, and again let $m$ denote the normalized Lebesgue measure in the unit disk $D$. Then $f$ is a signed q-function by Proposition [58, It is strictly increasing and positive. However, $f$ splits into the difference $2 x$ and $3 x^{2}-2 x^{3}$, where the latter is known to be a q-function which maps even $m$ to non-trivial topological measures (i.e. topological measures which are not restrictions of regular Borel measures). Hence $f_{*} m$ is a signed topological measure, but it is neither a topological measure nor a signed Borel measure. Indeed, let $C_{1}, C_{2} \in \mathcal{C}_{s}(D)$ be disjoint with $m\left(C_{1}\right)=\frac{1}{3}, m\left(C_{2}\right)=\frac{2}{5}$, and choose $C \in \mathcal{C}_{s}(D)$ with $C_{1} \cup C_{2} \subset C$ and $m(C)=\frac{4}{5}$. Then $\left(f_{*} m\right)(C)<\left(f_{*} m\right)\left(C_{1}\right)+\left(f_{*} m\right)\left(C_{2}\right)$, and consequently $f_{*} m$ is not a topological measure.

Before we embark on a total classification of the signed q-functions, we will need some notation for trees:

Notation 60. We will assume a rooted tree $T=(V, E)$ to be finite and directed, where $V$ is the set of vertices and $E$ is the set of edges. There is a level function $l: V \rightarrow \mathbb{N}$ where the root has level zero, vertices adjacent to the root has level one, and so forth. There are two maps $r, s: E \rightarrow V$ determined by $e=(s(e), r(e))$ $(e \in E)$. We call $r$ and $s$ the range and source map of $T$, respectively. 
Proposition 61. Let $X$ be any q-space and let $C \in \mathcal{C}_{f}$. Then there is a finite rooted tree $T=(V, E)$ (not necessarily unique) representing $C$ in the following sense:

(1) $\{A \in V: l(A)$ is an odd number $\} \subset \mathcal{C}_{s}$ and $\{A \in V: l(A)$ is even $\} \subset \mathcal{O}_{s}$.

(2) For each $e \in E$ we have $\biguplus_{A \in r(e)} A \subset s(e)$.

(3) Let $n=\max _{v \in V}\left\lceil\frac{l(v)}{2}\right\rceil$; then

$$
C=\biguplus_{i=0}^{n-1}\left(\biguplus_{l(K)=2 i+1}\left(K \backslash\left(\underset{V \in r\left(s^{-1}(K)\right)}{\biguplus^{\prime}} V\right)\right)\right) .
$$

Proof. Let $X$ be the root of $T$. Pick $x \in X \backslash C$ and for each $i$ let $j^{\prime}$ be determined by $x \in C_{i}^{j^{\prime}}$. Put $K_{i}=C_{i} \cup\left(\bigcup_{j \neq j^{\prime}} C_{i}^{j}\right)$. Then for $i \neq k$ we have $K_{i} \subset K_{k}$, $K_{k} \subset K_{i}$ or $K_{i} \cap K_{k}=\emptyset$. Let $r\left(s^{-1}(X)\right)$ be the sets $K_{i}, i=1,2, \ldots$, such that $K_{i} \backslash\left(\bigcup_{k \neq i} K_{k}\right) \neq \emptyset$. Now recursively for $K_{i} \in V \cap \mathcal{C}_{s}$ let $r\left(s^{-1}\left(K_{i}\right)\right)$ be the set $\left\{C_{i}^{j}\right\}_{j \neq j^{\prime}}$, and recursively for $C_{i}^{j}$ let

$$
r\left(s^{-1}\left(C_{i}^{j}\right)\right)=\left\{K_{k}: K_{k} \subset C_{i}^{j}, K_{i} \backslash\left(\bigcup_{\substack{k \neq i \\ K_{k} \subset C_{i}^{j}}} K_{k}\right) \neq \emptyset\right\} .
$$

It is now straightforward to verify the requirements of the tree $T$.

Remark 62. The construction also works for sets in $\mathcal{C}_{0}$ (which in general give infinite trees), in spaces of general genus. There is an alternative interpretation of the tree as unrooted, for which a vertex $V$ is relabeled with the set $V \backslash \biguplus_{W \in r\left(s^{-1}(V)\right)} W$, while each edge $e$ is labeled with the original $r(e)$ if $r(e)$ is open, and with $X \backslash r(e)$ otherwise; in the second case the direction of the edge is reversed. In this case the vertices are a partition of the space $X$, with the source vertices corresponding to the components of $C$, and the edges adjacent to a vertex correspond either to its co-complements or to their complements.

Definition 63. Let $T=(V, E)$ be a rooted tree with root $v_{0}$ and a function $w: V \rightarrow \mathbb{R}^{+}$. We call $T$ monotonic if $w\left(v_{0}\right)=1$ and

$$
w(v) \geq \sum_{v \in s(e)} r(e) \text { for all } v \in V,
$$

where the inequality is strict if $l(v)$ even.

Theorem 64. A function $f:[0,1] \rightarrow \mathbb{R}$ is a signed $q$-function if and only if the following hold:

(1) We have $f(0)=0$ and

$$
f\left(x^{-}\right)+f(1-x)=f(1) \text { for all } x \in(0,1] .
$$

(2) There is an $M \in \mathbb{R}^{+}$such that for any monotonic tree $(V, E, w)$ with root $v_{0}$ we have

$$
\left|\sum_{\{v \in V: l(v) \text { odd }\}} f(w(v))-\sum_{\left\{v \in V \backslash\left\{v_{0}\right\}: l(v) \text { even }\right\}} f\left(w(v)^{-}\right)\right| \leq M .
$$


Proof. Suppose $f$ is a function satisfying the requirements of the theorem. For any q-space $X$ define $f_{*}: Q_{1}(X) \rightarrow Q(X)$ according to Definition 54 Let $\mu \in Q_{1}(X)$ be arbitrary; we need to show that $f_{*} \mu$ is a signed solid set-function.

Since q-spaces only exhibit trivial partitions, the additivity requirement (Definition 43(3)) is satisfied by definition of $f_{*} \mu$ on $\mathcal{O}_{s}$. The regularity requirement (Definition 43(2)) of $f_{*} \mu$ follows from Theorem 64(1).

Let $C \in \mathcal{C}_{f}$, let $M$ be as in Theorem 64(2), and let $T=(V, E)$ be a tree representation of $C$ according to Proposition 61, with notation as in the proof of the proposition. Then $(V, E, \mu)$ is a monotonic tree. We obtain

$$
\begin{aligned}
& \left|\sum_{i}\left(\left(f_{*} \mu\right)(X)-\sum_{j}\left(f_{*} \mu\right)\left(C_{i}^{j}\right)\right)\right| \\
& =\left|\sum_{i}\left(f(1)-\sum_{j}\left(f\left(\mu\left(C_{i}^{j}\right)^{-}\right)\right)\right)\right| \\
& =\left|\sum_{i}\left(\left(f\left(\mu\left(K_{i}\right)\right)+f\left(\mu\left(C_{i}^{j^{\prime}}\right)^{-}\right)\right)-\sum_{j}\left(f\left(\mu\left(C_{i}^{j}\right)^{-}\right)\right)\right)\right| \\
& .=\left|\sum_{i}\left(f\left(\mu\left(K_{i}\right)\right)-\sum_{j \neq j^{\prime}}\left(f\left(\mu\left(C_{i}^{j}\right)^{-}\right)\right)\right)\right| \leq M .
\end{aligned}
$$

By Theorem 53 and Definition 43 it is now clear that $f$ is a signed q-function.

For the converse, assume that $f$ is a signed q-function. By Proposition 56 it is clear that $f\left(x^{-}\right)+f(1-x)=f(1)$ for all $x \in(0,1]$. Clearly, $f(0)=0$ since the empty set is a zero set.

It remains to prove Theorem 64 (2) for $f$. Let $m$ denote the Lebesgue measure in the unit square $I^{2}=[0,1] \times[0,1]$. Then $f_{*} m: \mathcal{A}_{s} \rightarrow \mathbb{R}$ is assumed to be a solid set-function, and in particular satisfying Definition 43(1) for some $M$. For any monotonic tree $(V, E, w)$ we may recursively construct a set $C \in \mathcal{C}_{f}\left(I^{2}\right)$ such that $(V, E)$ represents $C$ and $w \equiv m$. The set of vertices $V$ may even be taken to be rectangles. By the calculations above we obtain

$$
\begin{aligned}
& \left|\sum_{\{v \in V: l(v) \text { odd }\}} f(w(v))-\sum_{\left\{v \in V \backslash\left\{v_{0}\right\}: l(v) \text { even }\right\}} f\left(w(v)^{-}\right)\right| \\
& =\left|\sum_{i}\left(f\left(m\left(K_{i}\right)\right)-\sum_{j \neq j^{\prime}} f\left(m\left(C_{i}^{j}\right)^{-}\right)\right)\right| \\
& =\left|\sum_{i}\left(\left(f_{*} m\right)(X)-\sum_{j}\left(f_{*} m\right)\left(C_{i}^{j}\right)\right)\right| \leq M,
\end{aligned}
$$

and hence $f$ satisfies the requirements of the theorem. The proof is complete.

Corollary 65. Let $m$ be Lebesgue measure on the unit square $[0,1] \times[0,1]$, and let $f:[0,1] \rightarrow \mathbb{R}$. Then $f$ is a signed $q$-function if and only if $f_{*} m$ extends to a signed topological measure. 


\section{REFERENCES}

[1] J. F. Aarnes: Quasi-states and Quasi-measures, Adv. in Math. 86 (1991), 41-67. MR1097027 (92d:46152)

[2] J. F. Aarnes: Pure quasi-states and extremal quasi-measures, Math. Ann. 295 (1993), 575-588. MR.1214949 (94e:46096)

[3] J. F. Aarnes: Construction of non-subadditive measures and discretization of Borel measures, Fund. Math. 147 (1995), 213-237. MR1348720 (96k:28022)

[4] J. F. Aarnes and A. B. Rustad: Probability and quasi-measures - a new interpretation, Math. Scand. 85 (1999), 278-284. MR:1724240 (2001h:28015)

[5] S. Butler: Density in the space of topological measures, Fund. Math. (2002), no. 3, 239-251. MR.1925001 (2003f:28029)

[6] D. Grubb: Signed quasi-measures, Trans. Amer. Math. Soc. 349 (1997), no. 3, 1081-1089. MR:1407700 (98c:28012)

[7] D. Grubb: Signed quasi-measures and dimension theory, Proc. Amer. Soc. 128 (2000), no. 4, 1105-1108. MR.1636950 (2000i:28014)

[8] D. J. Grubb and T. LaBerge: Additivity of quasi-measures, Proc. Amer. Math. Soc. 126 (1998), no. 10, 3007-3012. MR1458874 (99a:28011)

[9] F. F. Knudsen: Topology and the construction of extreme quasi-measures, Adv. in Math. 120 (1996), 302-321. MR1397085 (97e:28007)

Department of Mathematical Sciences, Norwegian University of Science and TechNOLOGY, Trondheim, NORWAY

Department of Mathematical Sciences, Norwegian University of Science and TechNOLOGY, TRONDHEIM, NORWAY

Current address: Statoil Research Centre, N-7005 Trondheim, Norway 